\title{
Glicemia de ayuno versus prueba de tolerancia oral a la glucosa en la detección de intolerancia a la glucosa en niños y adolescentes obesos
}

\author{
Vivian Gallardo $\mathrm{T}^{1}$, Alejandra Avila $\mathrm{A}^{1,2, a}, \mathrm{~N}$ ancy U nuane $\mathrm{M}^{1}$, \\ Ethel Codner'. \\ Fasting glucose versus oral glucose \\ tolerance test for detection of glucose \\ intolerance in obese children
}

Background: Recently, the cut-off point for normal fasting glucose (FG) level, was decreased to $100 \mathrm{mg} / \mathrm{dl}$. Aim: To determine the frequency of abnormal carbohydrate abnormalities in children with obesity and evaluate if the fasting glucose level is a useful tool for the screening of glucose intolerance (GI). Patients and methods: Children and adolescents, referred for evaluation of obesity were evaluated with an oral glucose tolerance test (OGTT) and FG. The sensitivity of FG for detection of GI, using the 100 and $110 \mathrm{mg} / \mathrm{dl}$ cut-off point, was evaluated. Results: We studied 186 patients (125 females) aged 12.1 (range: 5.4-19.3) years with a body mass index (BMI) of 29.9 (18.3-44.6) $\mathrm{kg} / \mathrm{mt}^{2}$ and a BMI Z score of 2.1 (1.7-3.2). Seven patients (3.8\%) had abnormalities in the carbohydrate metabolism. The sensitivity of FG for the detection of GI using the 100 and $110 \mathrm{mg} / \mathrm{dl}$ cut-off values was 42.9 and 14.3\%, respectively. Receiver operating characteristic (ROC) curves showed that the optimal diagnostic level for FG corresponds to $80 \mathrm{mg} / \mathrm{dl}$ (sensitivity: $85.7 \%$ and specificity of $74.9 \%$ ). Conclusions: An abnormal carbohydrate metabolism was detected in $3.8 \%$ of the obese children and adolescents in this sample. FG of $100 \mathrm{mg} / \mathrm{dl}$ does not detect $57.1 \%$ of the patients with glucose intolerance. These data suggest that FG is not a useful screening tool for glucose intolerance in young patients (Rev Méd Chile 2006; 134: 1146-52).

(Key w ords: Blood glucose; Glucose tolerance test; Obesity; Diabetes Mellitus)

\begin{abstract}
Recibido el 31 de agosto, 2005. Aceptado el 14 de marzo, 2006.
Trabajo financiado parcialmente por el Proyecto Fondecyt \#1050452.

${ }^{1}$ Instituto de Investigaciones Materno Infantil (IDIMI), Facultad de Medicina, Universidad de Chile. ${ }^{2}$ Hospital Clínico San Borja Arriarán, Servicio de Salud Metropolitano Centro.

anfermera Universitaria.
\end{abstract}

os criterios diagnósticos para diabetes mellitus ـ(DM) e intolerancia a la glucosa (IG) han sufrido modificaciones a través del tiempo. Es así

Correspondencia a: Dra. Ethel Codner D. Instituto de Investigaciones Materno Infantil (IDIMI), Universidad de Chile. Casilla 226-3, Santiago, Chile. Teléfono: 562-4248280. Fax: 562-424-7240. E mail: ecodner@med.uchile.cl como en el año 1997 la Asociación Americana de Diabetes (ADA) disminuyó el valor de la glicemia de ayuno (GA) que realiza el diagnóstico de DM desde 140 a $126 \mathrm{mg} / \mathrm{dl}^{1}$. El principal motivo para efectuar este cambio fue la presencia de retinopatía diabética con valores de GA menores a lo establecido hasta ese momento ${ }^{2,3}$. En forma semejante, la Organización Mundial de la Salud en 
1999 (OMS 1999) también disminuyó el valor de glicemia normal a $125 \mathrm{mg} / \mathrm{dl}^{4}$.

En 2003, la ADA cambió nuevamente el límite normal de la GA, esta vez a $100 \mathrm{mg} / \mathrm{dl}^{5}$, basándose en estudios que demuestran que una glicemia de ayuno (GA) sobre $100 \mathrm{mg} / \mathrm{dl}$ ya predice diabetes en el futuro 6,7 , y que este valor normal de la GA se correlaciona mejor con una prueba de tolerancia oral a la glucosa (PTGO) alterada ${ }^{5,8}$. Además, la ADA crea la categoría de intolerancia de ayuno para los sujetos con GA alterada sin llegar a los límites de DM y sugiere que la GA es suficiente para clasificar a un sujeto. En cambio, la OMS 1999 no ha disminuido el valor normal de GA, y además recomienda realizar una PTGO en los sujetos con GA entre 110 y $125 \mathrm{mg} / \mathrm{dl}^{4}$. En la Tabla 1 se describen los criterios diagnósticos de DM e IG según ADA 1997, ADA 2003 y OMS 1999.

En los últimos 20 años, la incidencia de DM tipo 2 (DM2) en la población joven ha aumentado, incluso se ha descrito una epidemia de DM2 en los países desarrollados, asociada a la alta prevalencia de la obesidad infanto-juvenil ${ }^{9}$. En series en el extranjero se ha descrito una incidencia de intolerancia a la glucosa en niños y adolescentes obesos entre $4,5 \%$ en italianos ${ }^{10}$ y $22 \%$ en americanos $^{11}$. En nuestro país se ha descrito un aumento de la obesidad de $3,2 \%$ a $21,4 \%$ en la última década ${ }^{12}$, pero no hay publicaciones de la prevalencia de intolerancia a la glucosa en niños que se atienden en el sistema público.
Los objetivos de este estudio son determinar la prevalencia de alteración del metabolismo a los hidratos de carbono en niños y adolescentes obesos y evaluar si la disminución de la GA a 100 $\mathrm{mg} / \mathrm{dl}$ logra detectar a los niños y adolescentes que son intolerantes a la glucosa a las $2 \mathrm{~h}$ en la PTGO.

\section{MATERIALES Y MÉTODO}

Se estudiaron todos los niños obesos que consultaron al policlínico de Endocrinología Infantil del Hospital San Borja Arriarán (HCSBA) entre mayo de 2000 y abril de 2004.

Los pacientes fueron medidos en un estadiómetro de Harpenden ${ }^{\circledR}$ con precisión de 0,1 mm y pesados en balanza Seca ${ }^{\circledR}$ con precisión de 0,1 kg. La antropometría se analizó según la curva NCHS $2000^{13}$, definiendo como obesos a los pacientes con un índice de masa corporal (IMC) mayor al percentil 95 (1,65 desviaciones estándares), según las tablas de NCHS $2000^{11}$. El IMC se expresó como desviaciones estándares (DS) para la edad y sexo (puntaje Z). Se determinó presencia y severidad de acantosis nigricans y el estadio puberal según Tanner ${ }^{14,15}$. Se clasificó como prepuberal a las niñas con mamas Tanner 1 y a los varones con volumen testicular menor a $4 \mathrm{cc}$ usando orquidómetro de Prader.

A todos los pacientes se les realizó una PTGO con 1,75 grs de glucosa por kilo de peso (con un

Tabla 1. C riterios diagnósticos de diabetes mellitus, intolerancia de ayuno e intolerancia a glucosa

\begin{tabular}{|lccc|}
\hline & ADA 1997 & OMS 1999 & ADA 2003 \\
& Glicemia (mg/dl) & Glicemia (mg/dl) & Glicemia (mg/dl) \\
\hline $\begin{array}{l}\text { Glicemia de ayuno } \\
\text { Diabetes }\end{array}$ & $>125$ & $>125$ & $>125$ \\
Intolerancia de ayuno & $110-125$ & $*$ & $100-125$ \\
Prueba de tolerancia oral a la glucosa (2 h poscarga) & & $\geq 200$ & \\
$\quad$ Diabetes & $\geq 200$ & $140-199$ & $140-199$ \\
Intolerancia a la glucosa & $140-199$ & & $\geq 200$ \\
Glicemia en cualquier momento del día asociada a síntomas & $\geq 200$ & \\
Diabetes & $\geq 200$ & $\geq 200$ & \\
\hline
\end{tabular}

*: OMS 1999 recomienda realizar prueba de tolerancia oral a la glucosa si la glicemia de ayuno se encuentra entre $110-125 \mathrm{mg} / \mathrm{dl}$. 
máximo de 75 grs), midiendo glicemia (G) e insulinemia (I) basal y a los $120 \mathrm{~min}$. Las muestras fueron tomadas entre 8 y $10 \mathrm{AM}$, con al menos 10 $\mathrm{h}$ de ayuno y con un régimen de $300 \mathrm{~g}$ de hidratos de carbono (HC) durante los 3 días previos al examen. La glicemia fue medida en plasma con método de glucosa oxidasa y la insulinemia con método de radioinmunoensayo (Diagnostic Product Corporation, Los Angeles, CA), cuya coeficiente variación intraensayo e interensayo es de $5-9 \%$ y $5-10 \%$, respectivamente. Las muestras con insulinemias sobre $100 \mathrm{mU} / \mathrm{ml}$ se repitieron con la muestra diluida, y posteriormente fueron reconfirmadas.

Se clasificó al paciente como intolerante de ayuno (IA) si la glicemia de ayuno se encontraba entre 100 y $125 \mathrm{mg} / \mathrm{dl}$ (Criterio ADA 2003), y como intolerante a la glucosa (IG) si la glicemia a las $2 \mathrm{~h}$ en la PTGO se encontraba entre 140 y 199 $\mathrm{mg} / \mathrm{dl}$, como sugiere OMS 1999. También se evaluó la sensibilidad diagnóstica de la GA según criterio ADA 1997, utilizando GA 110-125 mg/dl (Tabla 2). Se determinó el número total de pacientes que presentó alguna alteración en el metabolismo de HC usando cualquiera de los 3 criterios (pacientes con alteración metabolismo de los hidratos de carbono»), y se evaluaron las características clínicas de ellos. Además, se estimó sensibilidad de la glicemia de ayuno para detectar a estos pacientes. También se realizó una curva ROC (receiver operator characteristics) para determinar el valor de GA con mayor sensibilidad y especificidad en detectar una PTGO alterada.

Se estimó la resistencia insulínica mediante el índice de HOMA-IR (Homeostasis model assesment index) mediante la siguiente fórmula: HOMA-IR $=$ (Glicemia basal $(\mathrm{mg} / \mathrm{dl}) \mathrm{x}$ insulinemia basal $(\mathrm{uU} / \mathrm{ml}) / 405]^{16}$. Se compararon las características clínicas y de laboratorio de los sujetos con y sin alteración metabolismo de los hidratos de carbono utilizando prueba U de Mann-Whitney y prueba exacta de Fisher. Se muestran los resultados como mediana y rangos. Se consideró como significativo un $\mathrm{p}<0,05$.

\section{RESUlTADOs}

Se reclutaron 186 pacientes (125 mujeres, 139 púberes) con una edad promedio de 12,1 (5,419,3) años, un IMC promedio de 29,9 (18,3-44,6) $\mathrm{kg} / \mathrm{mt}^{2}$, correspondiente a un puntaje $\mathrm{Z}$ de IMC de $2,1(1,7-3,2)$ DS. Las características clínicas de los sujetos estudiados se encuentran descritas en la Tabla 2.

De los 186 sujetos estudiados, 7 presentaron alteración del metabolismo de los hidratos de carbono (3,8\%), siendo la alteración más frecuente

Tabla 2. Características clínicas y de laboratorio del grupo total de pacientes estudiados, y en aquéllos con y sin alteración del metabolismo de los carbohidratos (HC). Los datos se muestran como mediana y rangos

\begin{tabular}{|lccc|}
\hline & Grupo total & $\begin{array}{c}\text { Pacientes sin alteración } \\
\text { metabolismo HC }\end{array}$ & $\begin{array}{c}\text { Pacientes con alteración } \\
\text { metabolismo HC }\end{array}$ \\
\hline $\mathrm{N}$ & 186 & 179 & 7 \\
Mujeres (\%) & $125(67,2)$ & $119(66,5)$ & $6(85,7)$ \\
Púberes (\%) & $139(74,7)$ & $133(74,3)$ & $6(85,7)$ \\
Edad & $12,1(5,4-19,3)$ & $12(5,4-19,3)$ & $13,9(7,3-15,8)$ \\
$\mathrm{IMC}\left(\mathrm{kg} / \mathrm{mt}^{2}\right)$ & $29,3(18,3-44,6)$ & $29,2(18,3-44,6)$ & $35(26,2-36,3)$ \\
IMC (Puntaje Z) & $2,1(1,7-3,2)$ & $2,1(1,7-3,2)$ & $2,4(1,9-2,5)$ \\
Glicemia ayuno (mg/dl) & $76(48-219)$ & $75(48-97)$ & $95(71-219)^{* *}$ \\
Glicemia 2 h PTGO (mg/dl) & $86(45-410)$ & $86(45-138)$ & $151(69-410)^{* *}$ \\
Insulinemia ayuno (uU/ml) & $25,7(3,7-131)$ & $24,8(3,7-101)$ & $54,7(6,8-131)^{*}$ \\
Insulinemia 2 h PTGO (uU/ml) & $85,6(6,4-493,2)$ & $79,7(6,4-493,2)$ & $219,8(10,8-389)^{*}$ \\
HOMA-IR & $5(0,7-59,1)$ & $4,7(0,7-59,1)$ & $12,8(7,6-29,4)^{* * *}$ \\
\hline
\end{tabular}

HC: Hidratos de carbonos. Los resultados se muestran como mediana (rango). * $p<0,05, *_{p}^{*}<0,01, * * *_{p}<0,001$ : Prueba U de Mann-Whitney. Comparación de pacientes con versus sin alteración del metabolismo de los carbohidratos. 
la IG (2,2\%). Las características clínicas de estos sujetos se presentan en la Tabla 3. Utilizando la GA de $100 \mathrm{mg} / \mathrm{dl}$ se diagnosticaron 3 pacientes con alteración en el metabolismo de hidratos de carbono, 1 caso de DM que se habría detectado con ambos puntos de corte y 2 casos de IA; si se hubiese utilizado una GA de $110 \mathrm{mg} / \mathrm{dl}$ sólo se habría diagnosticado el caso con DM. Por lo tanto, si no se realiza PTGO, como recomienda ADA 2003 y 1997, se habría detectado sólo 42,9 y $14,3 \%$, respectivamente, del total de pacientes con alteraciones en el metabolismo de los carbohidratos. En cambio, al utilizar el criterio OMS 1999 que exige usar PTGO, se habría pesquisado el mismo sujeto como DM y a otros cuatro como IG (sensibilidad: $71,4 \%$ ), pero no habrían sido diagnosticados dos sujetos con IA, cuya GA de ayuno estaba entre 100 y 110, y su PTGO resultó normal. La curva ROC (Figura 1) demostró que el punto con mayor utilidad diagnóstica de la GA se logra con el valor de $80 \mathrm{mg} / \mathrm{dl}$, el que tiene una sensibilidad de 85,7\% y una especificidad de $74,9 \%$ en la pesquisa de una PTGO alterada (área bajo la curva: $0,89, p=0,001$ ).

Todos los pacientes con IA e IG (pacientes $1 \mathrm{a}$ 6 en Tabla 3) eran puberales, tenían acantosis nigricans al examen físico y 2 eran mujeres con hiperandrogenismo e irregularidad menstrual compatibles con síndrome ovario poliquístico (Tabla 3). La paciente con DM fue detectada por las 3 clasificaciones, teniendo alterada tanto la glicemia de ayuno como la poscarga. Los pacientes con alteración del metabolismo de los hidratos de carbono presentaron mayores niveles de glicemia de ayuno y postcarga, insulinemia basal y estimulada, y HOMA-IR (Tabla 2).

\section{Discusión}

Este estudio demuestra una alteración del metabolismo de los carbohidratos en 3,8\% en niños y adolescentes obesos que consultan en un hospital público, frecuencia menor a la comunicada previamente en Estados Unidos de Norteamérica (USA) y en Chile, y similar a la de obesos italianos ${ }^{10}$. Sinha et al demostraron una prevalencia de intolerancia a la glucosa de $21 \%$ en los adolescentes obesos americanos ${ }^{11}$ y Barja et al publicaron una prevalencia de $11 \%$ en niños chilenos de un hospital privado con obesidad severa $(\text { IMC }>3 \mathrm{DS})^{17}$. Esta diferencia respecto a nuestros resultados puede estar dada por la menor magnitud del sobrepeso que presentaron los pacientes de nuestro trabajo, cuyo puntaje Z de IMC fue de 2,2 DS, en comparación con 4 DS observada en los otros grupos, diferencia que puede estar dada por disparidad en los criterios de selección de estas series ${ }^{11,17,18}$. Nuestro estudio demuestra que los pacientes infanto-juveniles que consultan por obesidad en un hospital chileno presentan obesidad de menor magnitud que

Tabla 3. Características de los pacientes con alteración del metabolismo de los hidratos de carbono

\begin{tabular}{|c|c|c|c|c|c|c|c|c|c|c|c|c|}
\hline \multirow{2}{*}{$\begin{array}{l}\text { Paciente } \\
\mathrm{N}^{\circ}\end{array}$} & \multirow{2}{*}{$\begin{array}{c}\text { Alteracion } \\
\text { HC }\end{array}$} & \multirow[t]{2}{*}{ Sexo } & \multirow{2}{*}{$\begin{array}{l}\text { Edad } \\
\text { (años) }\end{array}$} & \multirow[t]{2}{*}{ Tanner } & \multirow[t]{2}{*}{ Acantosis } & \multirow{2}{*}{$\underset{\left(\mathrm{kg} / \mathrm{mt}^{2}\right)}{\mathrm{IMC}}$} & \multirow{2}{*}{$\begin{array}{l}\text { Puntaje Z de } \\
\text { IMC (DS) }\end{array}$} & \multicolumn{2}{|c|}{ Glicemia } & \multicolumn{2}{|c|}{ Insulina } & \multirow[t]{2}{*}{ HOMA-IR } \\
\hline & & & & & & & & $\begin{array}{l}\text { Ayuno } \\
\text { (mg/dl) }\end{array}$ & $\begin{array}{c}2 \mathrm{hrs} \\
\text { PTG0 } \\
\text { (mg/dl) }\end{array}$ & $\begin{array}{l}\text { Ayuno } \\
(\mathrm{uU} / \mathrm{ml})\end{array}$ & $\begin{array}{c}2 \mathrm{hrs} \\
\text { PTGO } \\
\text { (uU/ml) }\end{array}$ & \\
\hline 1 & IG & F & 11,5 & 4 & H & 35,2 & 2,39 & 91 & 151 & 131 & 381 & 29,4 \\
\hline 2 & IG & F & 13,2 & 5 & H & 35,0 & 2,28 & 71 & 163 & 43,6 & 158,5 & 7,6 \\
\hline 3 & IG & $\mathrm{M}$ & 15,8 & 5 & + & 36,6 & 2,26 & 95 & 182 & 54,7 & 365 & 12,8 \\
\hline 4 & IG & F & 14,9 & 4 & H+ & 34,1 & 2,38 & 90 & 150 & 51,4 & 126 & 11,4 \\
\hline 5 & IA & $\mathrm{F}$ & 14,2 & 5 & H & 36,2 & 1,97 & 101 & 69 & 55,7 & 108,3 & 13,9 \\
\hline 6 & IA & $\mathrm{F}$ & 14,4 & 4 & + & 28,2 & 1,74 & 100 & 95 & 106 & 389 & 26,2 \\
\hline 7 & DM & $\mathrm{F}$ & 7,4 & 1 & ++ & 26,2 & 1,95 & 219 & 410 & 6,8 & 10,8 & 3,7 \\
\hline
\end{tabular}

HC: hidratos de carbono, IMC: Índice de masa corporal, PTGO: Prueba de tolerancia oral a la glucosa, IG: intolerancia a la glucosa, IA: intolerancia de ayuno, DM: diabetes mellitus. La severidad de la acantosis se describe según el número de cruces (t: leve, +t: moderada, ++t: severa). 
en otras series extranjeras. Otro factor que puede explicar la menor prevalencia en anormalidades del metabolismo de los carbohidratos podría corresponder a factores étnicos diferentes en los pacientes de hospital público y privado ${ }^{19}$.

La intolerancia a la glucosa fue la alteración del metabolismo de los carbohidratos más frecuentemente encontrada, ya que de los siete casos alterados, cuatro correspondieron a este diagnóstico. Estos pacientes presentaron GA de ayuno normal, y no habrían sido detectados si la PTGO no hubiese sido realizada. En cambio, sólo dos pacientes presentaron IA, con PTGO normal. Esta situación de mayor frecuencia de IG que de IA es opuesta a la encontrada en adultos, en que utilizando el punto de corte de $100 \mathrm{mg} / \mathrm{dl}$ se describe mayor frecuencia de IA que $\mathrm{IG}^{20}$. Una explicación a este fenómeno es que en la población joven predomina el fenómeno de insulinorresistencia sobre la deficiencia en la secreción de insulina por la célula $\aleph^{21,22}$, y la IG refleja fundamentalmente una situación de insulinorresistencia. La intolerancia de ayuno, en cambio, es signo de déficit de la secreción de esta hormo$n^{23}$, hecho fisiopatológico que se hace más importante a medida que el sujeto envejece.

Nuestros resultados demuestran que la disminución del valor de glicemia normal desde 110 a $100 \mathrm{mg} / \mathrm{dl}$, aumenta la sensibilidad en la pesquisa de alteración del metabolismo de los hidratos de carbono desde $14,3 \%$ a 42,9\%, pero aun así no hay concordancia entre los valores de GA y de PTGO. Esto queda demostrado por el hecho que los casos intolerantes de ayuno no presentaron intolerancia a la glucosa, y tampoco los IG fueron IA (Tabla 3). Más aún, la curva ROC demuestra que aun disminuyendo el punto de corte a $80 \mathrm{mg} /$ dl, sólo se detectaría el $85,7 \%$ de los casos con alteraciones (Tabla 3 y Figura 1), teniendo una baja especificidad, por lo que muchos sujetos sanos serían innecesariamente clasificados como en riesgo. Nuestros resultados son muy semejantes a los encontrados en adolescentes con síndrome de ovario poliquístico, otro grupo con riesgo de alteraciones del metabolismo de los carbohidratos, en que la curva ROC determinó valores semejantes de GA para detectar intolerancia a la glucosa que los observados en nuestro estudio ${ }^{24}$.

Recientemente, Davidson planteó que la PTGO sería superflua en la detección de alteracio- nes a los carbohidratos ${ }^{25}$, pero el bajo rendimiento diagnóstico de la GA que observamos en niños y adolescentes sugieren que éste no es un examen sensible para detectar las alteraciones en el metabolismo de los carbohidratos. El uso de este examen como única herramienta para pesquisar la intolerancia a la glucosa, como sugiere la ADA, implicaría perder una oportunidad de realizar tratamiento preventivo oportuno de la DM2 en el cuadro más frecuentemente observado en los adolescentes. Además, ante la detección de un caso de IA, aún no hay estudios claros que permitan asegurar qué conducta se debe seguir, ya que los beneficios de programas preventivos de DM2 han sido sólo evaluados en los pacientes con IG, pero no en los con $\mathrm{IA}^{26,27}$.

Nuestros resultados demuestran que las alteraciones del metabolismo de los carbohidratos, especialmente IG e IA, se presentan principalmente en sujetos puberales, ya que sólo 1 de los 47 pacientes prepuberales estudiados presentó glicemia alterada. Además, esta paciente presentó glicemia de ayuno $>200 \mathrm{mg} / \mathrm{dl}$, por lo que en ella no era necesario realizar una PTGO para clasificarla. Esto nos permite concluir que la PTGO es de bajo rendimiento en sujetos prepuberales, y sería de mayor utilidad para efectuar diagnóstico de intolerancia a la glucosa en

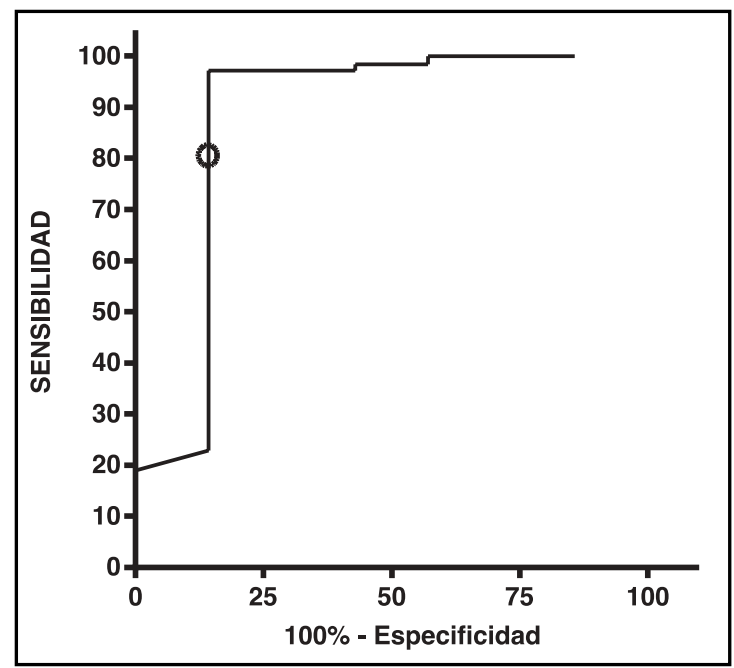

Figura 1. Curva ROC de glicemia de ayuno. El círculo muestra el punto con mayor sensibilidad y especificidad $(85,7$ y $74,9 \%$ respectivamente; área bajo la curva: $0,89, p=0,001$ ) 
sujetos obesos puberales o pospuberales. Estos resultados son semejantes a los descritos previamente por otros grupos ${ }^{28}$, y se explican por el fenómeno de insulinorresistencia fisiológico de la pubertad que se agrega al que ya existe en el paciente obeso 18,29 , razón por la que frecuentemente la DM2 del adolescente se inicia en la época peripuberal.

Recientemente la American Academy of Pediatris y la $\mathrm{ADA}^{30,31}$ sugirieron realizar pesquisa de DM2 en todos los sujetos obesos puberales con factores de riesgo realizando glicemia de ayuno cada dos años. Nuestros resultados, que demuestran una baja utilidad diagnóstica de la glicemia de ayuno, sugieren que este examen no cumple con el requisito fundamental exigido por la OMS, de tener una alta sensibilidad y especificidad para ser usado en un programa de detección de una patología ${ }^{32}$.

\section{REFERENCIAS}

1. Report of the Expert Committee on the Diagnosis and Classification of Diabetes Mellitus. Diabetes Care 1997; 20: 1183-97.

2. McCance DR, Hanson RL, Charles MA, Jacobsson LT, Pettitt DJ, Bennett PH et al. Comparison of tests for glycated haemoglobin and fasting and two hour plasma glucose concentrations as diagnostic methods for diabetes. BMJ 1994; 308: 1323-8.

3. Engelgau MM, Thompson TJ, Herman WH, Boyle JP, AuBERT RE, KeNNY SJ ET AL. Comparison of fasting and 2-hour glucose and HbA1c levels for diagnosing diabetes. Diagnostic criteria and performance revisited. Diabetes Care 1997; 20: 785-91.

4. Wordd Health Organization. Definition, Diagnosis and Classification of Diabetes Mellitus and its Complications: Report of a WHO Consultation. Part 1: Diagnosis and Classification of Diabetes Mellitus. Geneva: World Health Org. 1999.

5. Genuth S, Alberti KG, Bennett P, Buse J, Defronzo $\mathrm{R}$, KaHN R ET AL. Follow-up report on the diagnosis of diabetes mellitus. Diabetes Care 2003; 26 : 3160-7.

6. Gabir MM, Hanson RL, Dabelea D, Imperatore G, Roumain J, BenNett PH et al. The 1997 American Diabetes Association and 1999 World Health Organization criteria for hyperglycemia in the
Una de las limitaciones de este estudio es la ausencia de determinación de los niveles de glicemia de ayuno y PTGO en un grupo de niños y adolescentes con peso normal, pero estudios previos han demostrado que la glicemia en grupos sanos se mantiene estable 33 , y en general los estudios no han utilizado un grupo control sano para determinar la utilidad diagnóstica de esta prueba de laboratorio.

En conclusión, este estudio demuestra que existe $3,8 \%$ de alteración a los carbohidratos en niños y adolescentes con exceso de peso que consultan en un hospital público de Santiago, y que en este grupo continúa siendo necesaria realizar una PTGO para poder detectar IG. La disminución de la GA a 100 $\mathrm{mg} / \mathrm{dl}$ no logra reemplazar la PTGO, ya que tiene baja sensibilidad y baja concordancia con los valores obtenidos a las dos horas de la prueba de tolerancia oral a la glucosa.

diagnosis and prediction of diabetes. Diabetes Care 2000; 23: 1108-12.

7. Von Eckardstein A, Schulte H, Assmann G. Risk for diabetes mellitus in middle-aged Caucasian male participants of the PROCAM study: implications for the definition of impaired fasting glucose by the American Diabetes Association. Prospective Cardiovascular Munster. J Clin Endocrinol Metab 2000; 85: 3101-8.

8. Will new diagnostic criteria for diabetes mellitus change phenotype of patients with diabetes? Reanalysis of European epidemiological data. DECODE Study Group on behalf of the European Diabetes Epidemiology Study Group. BMJ 1998; 317: 371-5.

9. Rosenbloom AL, Joe JR, Young RS, Winter WE. Emerging epidemic of type 2 diabetes in youth. Diabetes Care 1999; 22: 345-54.

10. Invitti C, Guzzaloni G, Gilardini L, Moribato F, VIBERTI G. Prevalence and Concomitans of Glucosa Intolerante in European Obese Children and Adolescents. Diabetes Care 2003; 26: 118-24.

11. Sinha R, Fisch G, Teague B, Tamborlane WV, Banyas B, Auen K et al. Prevalence of impaired glucose tolerance among children and adolescents with marked obesity. N Engl J Med 2002; 346: 802-10.

12. Muzzo S, Burrows R, Cordero J, Ramírez I. Trends in nutritional status and stature among school-age 
children in Chile. Nutrition 2004; 20: 867-72.

13. Ogden CL, Kuczmarski RJ, Fiegal KM, Mei Z, Guo S, WEI R ET AL. Centers for Disease Control and Prevention 2000 growth charts for the United States: improvements to the 1977 National Center for Health Statistics version. Pediatrics 2002; 109: 45-60.

14. MarShall WA, TanNER JM. Variations in pattern of pubertal changes in girls. Arch Dis Child 1969; 44: 291-303.

15. Marshall WA, TANNER JM. Variations in the pattern of pubertal changes in boys. Arch Dis Child 1970; 45: 13-23.

16. Matthews DR, Hosker JP, Rudenski AS, Naylor BA, TREACHER DF, TurNER RC. Homeostasis model assessment: insulin resistance and beta-cell function from fasting plasma glucose and insulin concentrations in man. Diabetologia 1985; 28: 412-9.

17. Barja S, Hodgson MI, Acosta AM, Arteaga A. Intolerancia a la glucosa en niños obesos: comunicación preliminar. Rev Méd Chile 2003; 131: 419-26.

18. Weiss R, Dziura J, Burgert TS, Tamborlane WV, TAKSALI SE, YeCKEL CW ET AL. Obesity and the metabolic syndrome in children and adolescents. N Engl J Med 2004; 350: 2362-74.

19. Larenas G, Montecinos A, Manosalva M, Barthou M, VIDAL T. Incidence of insulin-dependent diabetes mellitus in the IX region of Chile: ethnic differences. Diabetes Res Clin Pract 1996; 34 Suppl: S147151.

20. Tai ES, Goh SY, Lee JJ, Wong MS, Heng D, Hughes $\mathrm{K}$ ET AL. Lowering the criterion for impaired fasting glucose: impact on disease prevalence and associated risk of diabetes and ischemic heart disease. Diabetes Care 2004; 27: 1728-34.

21. Weyer C, Bogardus C, Mott DM, Pratley RE. The natural history of insulin secretory dysfunction and insulin resistance in the pathogenesis of type 2 diabetes mellitus. J Clin Invest 1999; 104: 787-94.

22. Defronzo RA. Lilly lecture 1987. The triumvirate: beta-cell, muscle, liver. A collusion responsible for NIDDM. Diabetes 1988; 37: 667-87.

23. Carnevale Schianca GP, Rossi A, Sainaghi PP, Maduli E, BARTOL E. The significance of impaired fasting glucose versus impaired glucose tolerance: importance of insulin secretion and resistance. Diabetes Care 2003; 26: 1333-7.

24. Palmert MR, Gordon CM, Kartashov AI, Legro RS, Emans SJ, Dunaif A. Screening for abnormal glucose tolerance in adolescents with polycystic ovary syndrome. J Clin Endocrinol Metab 2002; 87: 1017-23.

25. Davidson MB. Counterpoint: The Oral Glucose Tolerance Test Is Superfluous. Diabetes Care 2002; 25: 1883-5.

26. Tuomilehto J, Lindstrom J, Eriksson JG, Vaue TT, Hamalainen $H$, Ilanne-Parikka P et al. Prevention of type 2 diabetes mellitus by changes in lifestyle among subjects with impaired glucose tolerance. N Engl J Med 2001; 344: 1343-50.

27. Knowier WC, Barrett-Connor E, Fowier SE, Hamman RF, LACHIN JM, WALKER EA ET AL. Reduction in the incidence of type 2 diabetes with lifestyle intervention or metformin. NEngl J Med 2002; 346: 393-403.

28. Dolan LM, Bean J, D'alessio D, Cohen RM, Morrison JA, Goodman E et al. Frequency of Abnormal Carbohydrate Metabolism and Diabetes in a Population-based Screening of Adolescents. Journal of Pediatrics, The 2005; 146: 751-8.

29. Morán A, Jacobs DR JR, Steinberger J, Hong CP, PRINEAS R, LUEPKER R ET AL. Insulin resistance during puberty: results from clamp studies in 357 children. Diabetes 1999; 48: 2039-44.

30. Type 2 diabetes in children and adolescents. American Diabetes Association. Pediatrics 2000; 105: 671-80.

31. American Diabetes Association. Type 2 diabetes in children and adolescents. Diabetes Care 2000; 23: 381-9.

32. WIISON J. Principles and practices for screening of diseases. Geneva: World Health Organization, 1968.

33. Wennlof AH, Yngve A, Nilsson TK, Sjostrom M. Serum lipids, glucose and insulin levels in healthy school children aged 9 and 15 years from Central Sweden: reference values in relation to biological, social and lifestyle factors. Scand J Clin Lab Invest 2005; 65: 65-76.

Agradecimientos

Agradecemos al Dr. Fernando Cassorla y al Dr. Néstor Soto por la cuidadosa revisión del manuscrito y a la Sra. Paulina Cohen por su apoyo con la medición de insulina. 